\title{
On reducing uncertainty on the Elliptical Plane modal identification method
}

\author{
Diogo Montalvão ${ }^{1, *}$, Mihai Dupac ${ }^{1}$,Daerefa-a Mitsheal Amafabia ${ }^{2}$, Opukuro David-West ${ }^{2}$ \\ and George Haritos \\ ${ }^{1}$ Department of Design and Engineering, Faculty of Science and Technology, Bournemouth \\ University, Poole House, Talbot Campus, Fern Barrow, Poole BH12 5BB, United Kingdom \\ ${ }^{2}$ School of Engineering and Technology, University of Hertfordshire, College Lane Campus, Hatfield \\ AL10 9AB, United Kingdom
}

\begin{abstract}
The Elliptical Plane has been recently introduced as a modal identification method that uses an alternative plot of the receptance. The method uses the dissipated energy per cycle of vibration as a starting point. For lightly damped systems with conveniently spaced modes, it produces quite accurate results, especially when compared to the well-known method of the inverse. When represented in the Elliptical Plane, the shape of the receptance is elliptical near resonant frequencies. The modal damping factor can be determined from the angle of the ellipse's major axis with the horizontal axis, whereas the real and imaginary parts of the modal constants can be determined from numerical curve-fitting (as in the method of the circle - Nyquist plot). However, the lack of points that can be used near the resonance (due to limitations in the frequency resolution, and effects from other modes near each resonance) and the fact that measurements are polluted by noise, bring uncertainty to the numerical curve-fitting. This paper aims at providing the first steps on the improvement of the quality of the modal identification of the receptance in the Elliptical Plane. The method and results are discussed with a multiple degree-of-freedom numerical example.
\end{abstract}

\section{Introduction}

The existing to date modal identification procedures cover different levels of sophistication. For example, the issue of determining the modal damping factors has recently been presented [1] from a different perspective, where the starting point was the dissipated energy per cycle of vibration rather than the governing equations of the dynamic motion. The proposed methodology was based on a special plot of the receptance, whereby the vertical axis is the sine of the phase angle and the horizontal axis is the amplitude. This plot has special properties, one of which is that the data points around a resonant frequency describe a loop that resembles the half of an ellipse [2]. For this reason, this special plot was named as the 'Elliptical Plane' of the receptance. It was also shown that the major and minor axis of the ellipse are related to the modal constants.

\footnotetext{
* Corresponding author: dmontalvao@bournemouth.ac.uk
} 
The Elliptical Plane method works quite well when in the presence of conveniently well-spaced mode shapes $[1,2]$, a common feature to other modal identification methods [3]. However, the determination of the modal properties in the Elliptical Plane is based on numerical extrapolation of the ellipse, which means that both the noise and the frequency resolution play a key role on the results. If different curve-fitting methods are used, results may be slightly different or can even fail by rendering straight lines or hyperbolas. Actually, in real scenarios where not many points are available, there can be a wide range of equally good solutions for the same problem.

\section{Theoretical development}

\subsection{Hysteretic damping in the Elliptical Plane}

It is possible to show that the energy dissipated per cycle of oscillation $W_{\text {diss }}$ for an oscillatory force with amplitude $F(\omega)$ for an angular frequency $\omega$ is given by the ellipse area of the force-displacement plot during a complete cycle [4, 5], which can be written as:

$$
W_{\text {diss }}=\pi h X^{2}(\omega)=\pi \eta k X^{2}(\omega)=\pi F(\omega) X(\omega) \sin [\theta(\omega)]
$$

where $k$ is the stiffness, $h$ is the damping coefficient, $X(\omega)$ is the amplitude of the displacement response, $\eta$ is the damping factor and $\theta(\omega)$ is the phase angle between the force and the response. From equation (1), and considering harmonic motion, the following relationship can be established:

$$
h \frac{X(\omega)}{F(\omega)}=\sin [\theta(\omega)]
$$

where the ratio $X(\omega) / F(\omega)$ is the amplitude $H(\omega)$ of the complex receptance $\alpha(\omega) \propto(\omega)$ (for harmonic motion). If we now drop the dependency on $\omega$ for a matter of convenience, Equation (2) can be re-written as:

$$
\sin (\theta)=h H
$$

Once the stiffness is known (which can be determined, e.g., from the method of the inverse [6]), the hysteretic damping factor can be determined from equation (1), i.e., $h=\eta k$. The determination of the damping factor and the generalisation to Multiple Degree-of-Freedom (MDOF) systems is addressed in detail in [1].

\subsection{Modal constants in the Elliptical Plane}

If the modes are sufficiently spaced in the frequency domain, and at the vicinity of a resonance $\omega_{r}$, the influence from other modes is small when compared to the resonant mode [3]. Therefore, the receptance of a MDOF system can be approximated by:

$$
\alpha_{\omega \rightarrow \omega_{r}} \cong \frac{A_{R}+i A_{I}}{\omega_{r}^{2}-\omega^{2}+i \eta_{r} \omega_{r}^{2}}
$$

where $A_{R}$ and $A_{I}$ are the real and imaginary parts of the modal constant, respectively, and $\eta_{r}$ and $\omega_{r}$ are the hysteretic damping factor and angular natural frequency, respectively, 
for mode $r, N$ is the number of Degrees-of-Freedom (DOFs) and $i=\sqrt{-1}$. Equation (4) resembles the equation of a Single DOF (SDOF) system with a complex modal constant.

Away from the natural frequency (i.e., $\left|\omega_{r}^{2}-\omega_{2}\right| \gg 0$ ), and considering, for better convenience, a lightly damped system where $\eta_{r} \cong 0$, equation (4) can be simplified to:

$$
\alpha_{\omega \ll \omega_{r}} \cong \frac{A_{R}+i A_{I}}{\omega_{r}^{2}-\omega^{2}}=\frac{A_{R}}{\omega_{r}^{2}-\omega^{2}}+i \frac{A_{I}}{\omega_{r}^{2}-\omega^{2}}
$$

If the receptance is represented in the Argand plane, then the phase $\theta_{\omega \ll \omega_{r}}$ is related to the imaginary $\alpha_{I_{\omega<\infty \omega_{r}}}$ and real $\alpha_{R_{\omega<\infty<\sigma_{r}}}$ parts of the receptance. Thus, in the $x \equiv H v S$ $y \equiv \sin (\theta)$ plane this becomes:

$$
\sin \left(\theta_{\omega \ll \omega_{r}}\right)=\sin \left[\tan ^{-1}\left(\frac{A_{I}}{A_{R}}\right)\right]
$$

When at the natural frequency, i.e., when $\omega=\omega_{y}$, equation (4) achieves its maximum value and the amplitude of the receptance at the resonance is:

$$
H_{\omega=\omega_{r}}=\left|\alpha_{\omega=\omega_{r}}\right|=\frac{1}{\eta_{r} \omega_{r}^{2}} \sqrt{A_{I}^{2}+A_{R}^{2}}
$$

which, when solved for $A_{R}$, becomes:

$$
A_{R}=\sqrt{H_{\omega=\omega_{r}}^{2} \eta_{r}^{2} \omega_{r}^{4}-A_{I}^{2}}
$$

If one solves equation (6) for $A_{I}$ when having equation (8) in consideration, and after some mathematical manipulation, this results in:

$$
A_{I}=\sqrt{\frac{H_{\omega=\omega_{r}}^{2} \eta_{r}^{2} \omega_{r}^{4}}{\left\{\tan \left[\sin ^{-1}\left(\theta_{\omega \ll \omega_{r}}\right)\right]\right\}^{2}+1}}
$$

\subsection{Numerical curve-fitting methods}

An ellipse as a particular case of conic can be described [7, 8] by the scalar product between the vector of coefficients $\mathbf{c}=\{a, b, c, d, e, f\}$ and the vector $\mathbf{v}=\left\{x, x y, y^{2}, x, y\right\}$ by:

$$
F_{\mathrm{c}}(\mathbf{v})=\mathbf{c} \cdot \mathbf{v}=a x+b x y+c y^{2}+d x+e y+f=0
$$

subject to $b^{2}<4 a c$. The function $F_{\mathbf{c}}(\mathbf{v})=F(x, y)$ represents the algebraic distance from a point $(x, y)$ to the conic. The fitting problem [9] can be approached by the use of a least square fitting (LSF) method based on the minimisation of the sum of the squared algebraic distances of the points $\left(x_{i}, y_{i}\right)$ to the conic:

$$
D_{1}(\mathbf{c})=\min _{\mathrm{c}} \sum_{k=1}^{n} F\left(x_{i}, y_{i}\right)^{2}=\min _{\mathrm{c}} \sum_{k=1}^{n} F_{\mathrm{c}}\left(\mathbf{v}_{\mathbf{i}}\right)^{2}=\min _{\mathrm{c}} \sum_{k=1}^{n}\left(\mathbf{c} \cdot \mathbf{v}_{\mathbf{i}}\right)^{2}
$$


with either $a+c=1 \quad[10], \quad a^{2}+\frac{1}{2} b+c^{2}=1 \quad$ [7], or $\quad\left\|x-c_{1}\right\|+\left\|x-c_{2}\right\|=2 a$

where $c_{1}$ and $c_{2}$ are the two foci of the ellipse and the norm in this case is calculated using a Mahalanobis distance. An iterative approximation method [7] through a better estimated geometric distance can be expressed by either one of the following two:

$$
D_{2}(\mathbf{c})=\sum_{k=1}^{n} \frac{F\left(\mathbf{c} \cdot \mathbf{v}_{\mathbf{i}}\right)^{2}}{\left\|\nabla_{\mathrm{x}} F\left(\mathbf{c} \cdot \mathbf{v}_{\mathbf{i}}\right)\right\|^{2}}[12] \text { or } D_{3}(\mathbf{c}) \cong \frac{\sum_{i=1}^{n} F\left(\mathbf{c} \cdot \mathbf{v}_{\mathbf{i}}\right)^{2}}{\sum_{k=1}^{n}\left\|\nabla_{\mathrm{x}} F\left(\mathbf{c} \cdot \mathbf{v}_{\mathbf{i}}\right)\right\|^{2}}[13]
$$

The approach in $[8,14]$ replaces the constraint $b^{2}<4 a c$ by the equality $b^{2}+1=4 a c$ and reformulate the fitting problem as:

$$
\min _{\mathbf{c}}\|\mathbf{H c}\|^{2} \quad \text { subject to } \quad \mathbf{c}^{\mathbf{T}} \mathbf{D c}=\mathbf{1}
$$

where the matrices $\mathbf{H}$ and $\mathbf{D}$ are expressed as in [14] by:

$$
\mathbf{H}=\left(\begin{array}{cccccc}
x_{1}^{2} & x_{1} y_{1} & y_{1}^{2} & x_{1} & y_{1} & 1 \\
\vdots & \vdots & \vdots & \vdots & \vdots & \vdots \\
x_{k}^{2} & x_{k} y_{k} & y_{k}^{2} & x_{k} & y_{k} & 1 \\
\vdots & \vdots & \vdots & \vdots & \vdots & \vdots \\
x_{n}^{2} & x_{n} y_{n} & y_{n}^{2} & x_{n} & y_{n} & 1
\end{array}\right) \text { and } \mathbf{D}=\left(\begin{array}{cccccc}
0 & 0 & 2 & 0 & 0 & 0 \\
0 & -1 & 0 & 0 & 0 & 0 \\
2 & 0 & 0 & 0 & 0 & 0 \\
0 & 0 & 0 & 0 & 0 & 0 \\
0 & 0 & 0 & 0 & 0 & 0 \\
0 & 0 & 0 & 0 & 0 & 0
\end{array}\right)
$$

\section{Numerical example}

A 2-DOF numerical model (a case from [2] with the theoretical properties shown in table 1) was used to test how the improved numerical curve-fitting method perform. The Elliptical Plane modal identification method [2] procedure assumes that the mode shapes are conveniently spaced, so that the influence from mode shapes at the vicinity of the mode shape being identified is as little as possible to be neglected.

Table 1. Numerical model's theoretical properties.

\begin{tabular}{|c|c|c|c|c|}
\hline $\begin{array}{c}\text { Mode } \\
\text { number }\end{array}$ & $A_{R}$ & $A_{I}$ & $\eta_{r}(\%)$ & Amplitude \\
\hline $1(20.4 \mathrm{~Hz})$ & 1000 & -500 & 1 & 6.805 \\
\hline $2(50.25 \mathrm{~Hz})$ & 2000 & -1200 & 5 & 0.4679 \\
\hline
\end{tabular}

When represented in the Elliptical Plane with $x \equiv H$ vs $y \equiv \sin (\theta)$ axes, the receptance takes an elliptical form near the resonant frequencies (figure 1) [2]. Since different methods may result in distinct best-fitting ellipses or even hyperbolas when "bad/noisy" data is used, the uncertainty in the modal identification can be only addressed by using the most adequate method. Restricting the best fit to the horizontal limits -1 and 1 (due to the properties of the Elliptical Plane described in [2]), this can be seen in figure 2 where distinct best-fitting ellipses have been obtained for the two sets of data from two 
different fitting methods, namely: the least squares method without any weights [9] presented in equation (11); and, the computationally efficient (and robust to noise) direct approach [14] shown in equation (13).

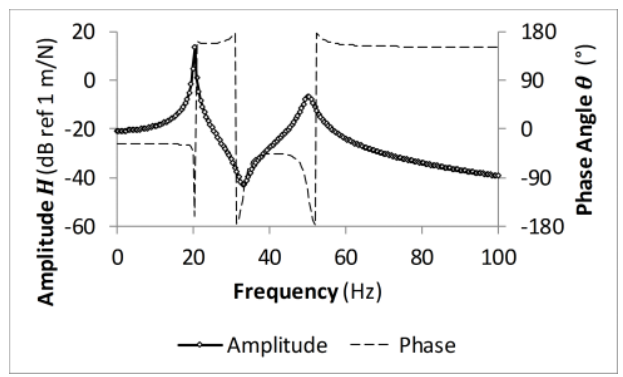

(a)

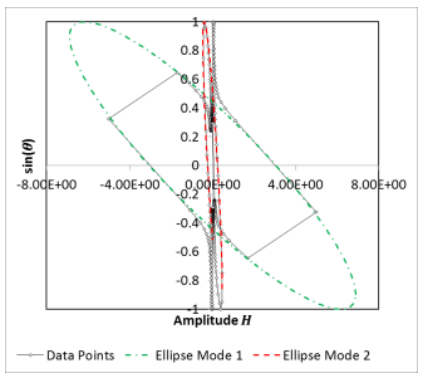

(b)

Figure 1. Amplitude and phase of the 2-DOF receptance case in table 1: (a) frequency domain; (b) Elliptical Plane

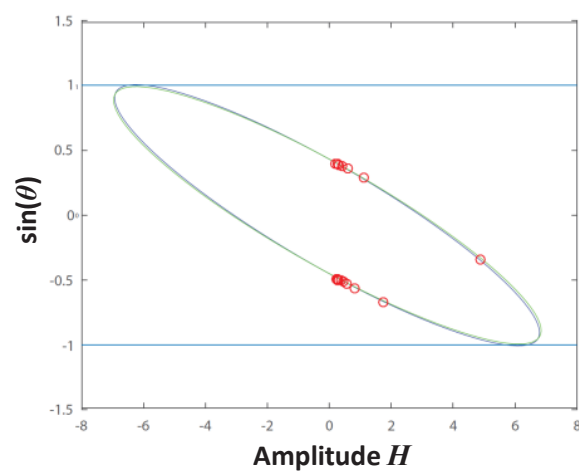

— LSF _ iterative LSF

(a)

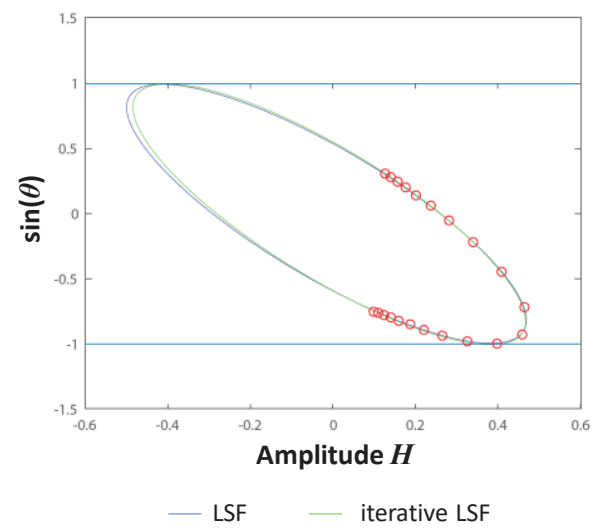

(b)

Figure 2. Best-fitting ellipses using the LSF method in equation (11) and the iterative method in equation (13): (a) data set 1 , and (b) data set 2 .

As a general rule, different methods such as conic residual or proximity methods render different ellipses. Independent of the methods used, almost elliptical distribution of the data points generate good and similar fits $[8,15]$, while a more noisy distribution may render different sets of ellipses or can even fail by rendering straight lines or hyperbolas [8]. In this case, since data is simulated and has no added noise, both methods produced very similar results. The results obtained from both methods were averaged and are shown in table 2 .

Table 2. Averaged numerical results from the modal identification in the $15-25 \mathrm{~Hz}$ and $45-55 \mathrm{~Hz}$ ranges (values between brackets are the percent error committed).

\begin{tabular}{|c|c|c|c|c|}
\hline $\begin{array}{c}\text { Mode } \\
\text { number }\end{array}$ & $A_{R}$ & $A_{I}$ & $\eta_{r}(\%)$ & Amplitude \\
\hline $1(20.4 \mathrm{~Hz})$ & $\begin{array}{c}994.6 \\
(\mathrm{e}=0.5 \%)\end{array}$ & $\begin{array}{c}-468.2 \\
(\mathrm{e}=6.4 \%)\end{array}$ & $\begin{array}{c}0.9769 \\
(\mathrm{e}=2.3 \%)\end{array}$ & $\begin{array}{c}6.849 \\
(\mathrm{e}=0.6 \%)\end{array}$ \\
\hline $2(50.25 \mathrm{~Hz})$ & $\begin{array}{c}2322 \\
(\mathrm{e}=16.1 \%)\end{array}$ & $\begin{array}{c}-1424 \\
(\mathrm{e}=18.7 \%)\end{array}$ & $\begin{array}{c}6.484 \\
(\mathrm{e}=29.7 \%)\end{array}$ & $\begin{array}{c}0.4215 \\
(\mathrm{e}=9.9 \%)\end{array}$ \\
\hline
\end{tabular}


Comparison of table 2 with results obtained in [2] for the same data points, show that, in general, the combination of the two methods introduced in this paper generally contributed to the improvement of the results, with a reduction on the average error from $19.1 \%$ to $10.5 \%$. It is important to that only one modal identification iteration was conducted.

\section{Conclusions}

The Elliptical plane method recently proposed for modal identification from the receptance, presented questions with regards with the quality of the numerical curve-fitting being used. In this paper, a combination of a least squares fitting (LSF) using a Mahalanobis distance and an iterative approximation method was explored. It is shown that there may be multiple solutions for the same problem, especially when the data points are sparse. However, the approach allows determining a range of values, which means that we can take the 'answer in the middle' as the solution to our problem. For future work, besides incorporating the possibility of the modal identification approach to be iterative (as in [2]), noisy data and experimental results will be used. Since different curve-fitting methods may yield different results, it will become appropriate to quantify confidence intervals rather than single point solutions. Also, since the elliptical shape of the receptance (in the vicinity of a resonant frequency) when represented in the 'Elliptical Plane' depends on both local and global modal properties, then it is reasonable to assume that this representation of the FRF can be used in other fields, such as in Structural Health Monitoring.

\section{References}

[1] D. Montalvão, J.M.M. Silva, Mechanical Systems and Signal Processing, 54-55 (2015)

[2] D. Montalvão, D.M. Amafabia, Mechanical systems and signal processing, 103 (2018)

[3] N.M.M. Maia, J.M.M. e Silva, Theoretical and experimental modal analysis (Research Studies Press, Taunton, 1997).

[4] B.J. Lazan, Damping of materials and members in structural mechanics (Pergamon Press Ltd., Oxford, 1968).

[5] D. Montalvão, R.A.L.D. Cláudio, A.M.R. Ribeiro, J. Duarte-Silva, Composite Structures, 97 (2013)

[6] D.J. Ewins, Modal testing: theory and practice, (Research studies Press, Letchworth, 1984).

[7] F.L. Bookstein, Computer Graphics and Image Processing, 9, 1 (1979)

[8] A. Fitzgibbon, M. Pilu, R.B. Fisher, IEEE Transactions on pattern analysis and machine intelligence, 21, 5 (1999)

[9] R.M. Haralick, L.G. Shapiro, Computer and robot vision, (Addison-wesley, 1992).

[10]W. Gander, G.H. Golub, R. Strebel, BIT Numerical Mathematics, 34, 4 (1994)

[11]J. Yu, S.R. Kulkarni, H.V. Pattern Recognition Letters, 33, 5 (2012)

[12]P.D. Sampson, Computer graphics and image processing, 18, 1 (1982)

[13]G. Taubin, IEEE Transactions on Pattern Analysis \& Machine Intelligence, 11 (1991)

[14]R. Halır, J. Flusser, editors. Proceedings of the $6^{\text {th }}$ International Conference in Central Europe on Computer Graphics and Visualization WSCG, 1998.

[15]K. Kanatani, P. Rangarajan, Computational Statistics \& Data Analysis, 55, 6 (2011). 